\title{
Thickness dependence of intrinsic dielectric response and apparent interfacial capacitance in ferroelectric thin films
}

N. A. Pertsev, R. Dittmann, R. Plonka, and R. Waser

Citation: Journal of Applied Physics 101, 074102 (2007);

View online: https://doi.org/10.1063/1.2713934

View Table of Contents: http://aip.scitation.org/toc/jap/101/7

Published by the American Institute of Physics

\section{Articles you may be interested in}

Ferroelectric thin films: Review of materials, properties, and applications

Journal of Applied Physics 100, 051606 (2006); 10.1063/1.2336999

Critical thickness of ultrathin ferroelectric $\mathrm{BaTiO}_{3}$ films

Applied Physics Letters 86, 102907 (2005); 10.1063/1.1880443

Depolarization fields in thin ferroelectric films

Journal of Applied Physics 44, 3379 (2003); 10.1063/1.1662770

Thickness-dependent dielectric constants of $(\mathrm{Ba}, \mathrm{Sr}) \mathrm{TiO}_{3}$ thin films with Pt or conducting oxide electrodes Journal of Applied Physics 92, 432 (2002); 10.1063/1.1483105

Thickness dependence of the effective dielectric constant in a thin film capacitor

Applied Physics Letters 73, 632 (1998); 10.1063/1.121930

Direct and indirect measurements on electrocaloric effect: Recent developments and perspectives Applied Physics Reviews 3, 031102 (2016); 10.1063/1.4958327

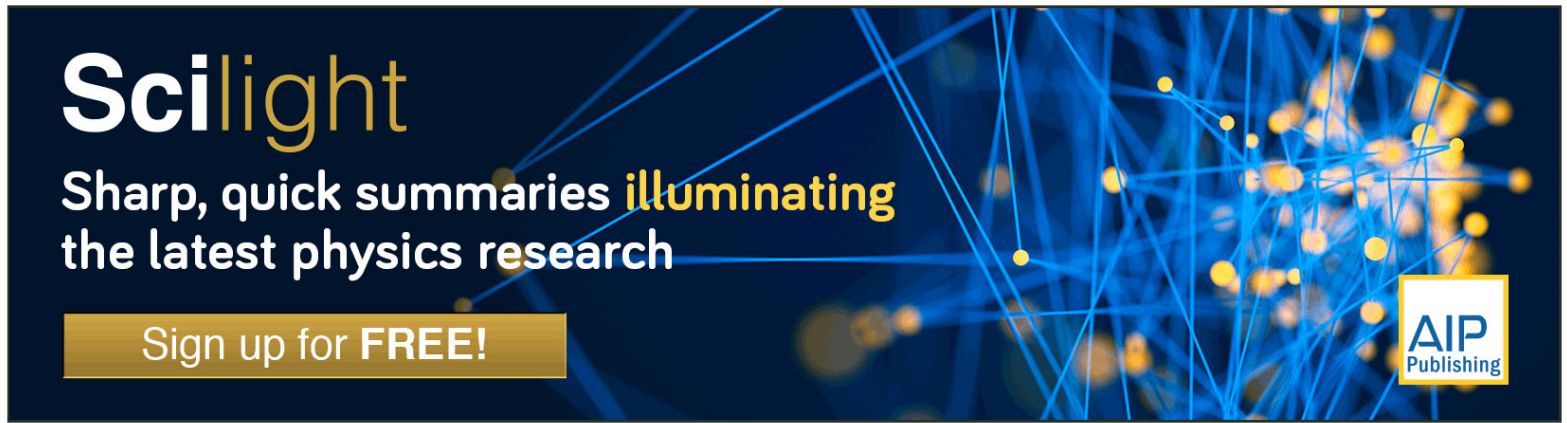




\title{
Thickness dependence of intrinsic dielectric response and apparent interfacial capacitance in ferroelectric thin films
}

\author{
N. A. Pertsever) and R. Dittmann \\ Institut für Festkörperforschung and CNI, Forschungszentrum Jülich, D-52425 Jülich, Germany \\ R. Plonka \\ Institut für Werkstoffe der Elektrotechnik, RWTH Aachen University, D-52056 Aachen, Germany \\ R. Waser \\ Institut für Festkörperforschung and CNI, Forschungszentrum Jülich, D-52425 Jülich \\ and Institut für Werkstoffe der Elektrotechnik, RWTH Aachen University, D-52056 Aachen, Germany
}

(Received 24 November 2006; accepted 21 January 2007; published online 3 April 2007)

\begin{abstract}
We studied theoretically the influence of the progressive strain relaxation and the depolarizing-field effect on the thickness dependence of the out-of-plane dielectric response of epitaxial ferroelectric thin films sandwiched between extended metal electrodes. The calculations show that the inverse of the measured capacitance varies with the film thickness almost linearly in the most part of the thickness range at the majority of temperatures. Extrapolation of this linear dependence to zero thickness usually gives considerable nonzero intercept even in the absence of nonferroelectric interfacial layers. Remarkably, such apparent "interfacial capacitance" in a certain temperature range becomes negative. The physical meaning of the effective dielectric constant, which can be extracted from the slope of the reciprocal capacitance thickness dependence, is also analyzed. The theoretical predictions are compared with the experimental data obtained for single-crystalline $\mathrm{SrRuO}_{3} / \mathrm{Ba}_{0.7} \mathrm{Sr}_{0.3} \mathrm{TiO}_{3} / \mathrm{SrRuO}_{3}$ and $\mathrm{Pt} / \mathrm{Ba}_{0.7} \mathrm{Sr}_{0.3} \mathrm{TiO}_{3} / \mathrm{SrRuO}_{3}$ thin-film capacitors. (C) 2007 American Institute of Physics. [DOI: 10.1063/1.2713934]
\end{abstract}

\section{INTRODUCTION}

Pronounced thickness dependence of the physical properties of ferroelectric thin films is a severe obstacle for the application of these materials in electronic devices which meet the demands of the current trend for miniaturization. Indeed, many groups reported that, as the film thickness is reduced, the dielectric response collapses, ${ }^{1,2}$ the remnant polarization decreases, ${ }^{3,4}$ while the coercive field increases. ${ }^{5,6}$ Even though very large permittivities $\sim 10000$ can be obtained for the bulk $\mathrm{Ba}_{0.7} \mathrm{Sr}_{0.3} \mathrm{TiO}_{3}$ material, ${ }^{7}$ its dielectric response in the thin-film form is suppressed by at least one order of magnitude ${ }^{1,2}$ and decreases further with decreasing thickness, which renders this material less attractive for using as a high-permittivity layer in future dynamic random access memories. ${ }^{8}$

The thickness dependence of the out-of-plane dielectric response measured in a conventional plate-capacitor setup was studied in many experimental and theoretical papers. Experimentally, it was revealed that the inverse of the measured capacitance varies linearly with the film thickness. ${ }^{1,2,9-15}$ This observation led to a widely accepted interpretation of the experimental data in terms of a "series capacitor model." According to such model, the measured capacitance density $c_{t}$ can be described by the relation

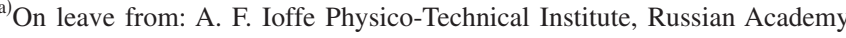
of Sciences, 194021 St. Petersburg, Russia; electronic mail: pertsev@domain.ioffe.ru
}

$$
\frac{1}{c_{t}}=\frac{1}{c_{i 1}}+\frac{t}{\varepsilon_{0} \varepsilon_{b}}+\frac{1}{c_{i 2}}
$$

where $\varepsilon_{b}$ denotes the relative permittivity of the bulk-like film interior, $\varepsilon_{0}$ is the permittivity of the vacuum, $t$ is the film thickness, and $c_{i 1}$ and $c_{i 2}$ are the capacitance densities associated with two film/electrode interfaces. The simplest possible explanation of finite interfacial capacitances $c_{i 1}$ and $c_{i 2}$ is the presence of low-permittivity layers at the film surfaces. Such a "dead" layer not necessarily has a different microstructure (e.g., due to reconstruction or high density of defects), because the intrinsic surface effect on the polarization ${ }^{16}$ may also reduce the local dielectric permittivity. ${ }^{17,18}$ The existence of a built-in electric field at the film/electrode interface represents another possible origin of suppressed dielectric response in the subsurface layer. ${ }^{2,18}$ On the other hand, the interfacial capacitance may mostly originate in the finite electronic screening length of metallic electrodes. ${ }^{19,20}$ This would be in agreement with the results of recent first-principles calculations ${ }^{21}$ and the latest experimental data on high quality $\mathrm{BaTiO}_{3}$ thin films, ${ }^{4}$ which indicate that the observed polarization relaxation can be attributed to the depolarizing field determined solely by imperfect compensation of ferroelectric polarization charges by electron density variations in $\mathrm{SrRuO}_{3}$ electrodes.

The form of Eq. (1) implies that the permittivity $\varepsilon_{b}$ of the film interior and the interfacial capacitances $c_{i 1}$ and $c_{i 2}$ are thickness-independent quantities. ${ }^{11}$ This assumption, however, seems to be generally incorrect for real ferroelectric capacitors. Indeed, several groups reported on the observation of a thickness-dependent phase transition 
temperature. ${ }^{11,15,22,23}$ Moreover, the intrinsic film permittivity is expected to vary with thickness at least for the following two reasons. First, the dielectric response of epitaxial films depends on the substrate-induced lattice strains, ${ }^{24}$ which relax as the thickness increases due to the generation of misfit dislocations. $^{25,26}$ Second, the depolarizing field induced by the polarization charges leads to a thickness-dependent shift of the ferroelectric transition temperature. ${ }^{27}$ Such shift changes the film permittivity and also makes it thickness dependent. ${ }^{15}$ We have shown recently that variations of the intrinsic dielectric response caused by these strain and depolarizing-field effects usually do not break a linear relationship between the reciprocal capacitance $c_{t}^{-1}$ and the film thickness. ${ }^{14,15}$ At the same time, our theoretical model predicts that such variations may change the constant term in Eq. (1) drastically.

In this article, we use a nonlinear thermodynamic theory to study in detail the influence of strain relaxation and depolarizing field on the thickness dependence of the small-signal dielectric response of ferroelectric capacitors. Our analysis is focused on epitaxial films of perovskite ferroelectrics grown on cubic substrates and sandwiched between continuous metal electrodes. The theoretical predictions are compared with the results of dielectric measurements carried out for $\mathrm{SrRuO}_{3} / \mathrm{Ba}_{0.7} \mathrm{Sr}_{0.3} \mathrm{TiO}_{3} / \mathrm{SrRuO}_{3}$ and $\mathrm{Pt} / \mathrm{Ba}_{0.7} \mathrm{Sr}_{0.3} \mathrm{TiO}_{3} / \mathrm{SrRuO}_{3}$ thin-film capacitors grown on $\mathrm{SrTiO}_{3}$ single crystals.

\section{THICKNESS DEPENDENCE OF PERMITTIVITY CAUSED BY STRAIN RELAXATION}

In this section, we focus on the thickness dependence of the dielectric properties that results from variations of lattice strains in epitaxial films, assuming that the depolarizing field in the ferroelectric capacitor is negligible. The strain effect on the film permittivity is closely related to the straininduced shift of the paraelectric to ferroelectric phase transition. According to the nonlinear thermodynamic theory of epitaxial ferroelectric films, ${ }^{24,28}$ the misfit strain $S_{m}$ in the film/substrate system has a strong impact on the transition temperature $T_{c}$. The stability range of the paraelectric phase in the "misfit strain-temperature" plane is defined by the condition $S_{m}^{*}(T)<S_{m}<S_{m}^{* *}(T)$, where $S_{m}^{*}$ and $S_{m}^{* *}$ are the critical misfit strains, at which the paraelectric phase becomes unstable with respect to the appearance of the out-of-plane and in-plane polarization components, respectively. These critical strains can be calculated as ${ }^{29}$

$$
\begin{aligned}
& S_{m}^{*}(T)=\frac{\left(s_{11}+s_{12}\right)}{4 \varepsilon_{0} C Q_{12}}(T-\theta), \\
& S_{m}^{* *}(T)=\frac{\left(s_{11}+s_{12}\right)}{2 \varepsilon_{0} C\left(Q_{11}+Q_{12}\right)}(T-\theta),
\end{aligned}
$$

where $\theta$ and $C$ are the Curie-Weiss temperature and constant of a stress-free bulk crystal, $Q_{m n}$ are the electrostrictive constants of paraelectric phase, and $s_{m n}$ are the film elastic compliances at constant polarization. In the temperature range $T>\theta$, Eqs. (2) and (3) describe the boundaries of a triangular region, where the paraelectric phase remains stable. It should be noted that perovskite ferroelectrics like $\mathrm{BaTiO}_{3}$, $\left(\mathrm{Ba}_{x} \mathrm{Sr}_{1-x}\right) \mathrm{TiO}_{3}, \mathrm{PbTiO}_{3}$, and $\mathrm{Pb}\left(\mathrm{Zr}_{1-x} \mathrm{Ti}_{x}\right) \mathrm{O}_{3}$, which are considered in this paper, have $Q_{12}<0$ and $Q_{11}+Q_{12}>0$. Therefore, the critical strain $S_{m}^{*}(T>\theta)$ is negative, whereas $S_{m}^{* *}(T$ $>\theta)$ is positive.

\section{A. Paraelectric regime}

Within the stability range of the paraelectric phase $\left(S_{m}^{*}\right.$ $<S_{m}<S_{m}^{* *}$ ), the relative out-of-plane permittivity $\varepsilon_{33}$ of an epitaxial film can be calculated as

$$
\varepsilon_{33}=\frac{K_{s}}{S_{m}-S_{m}^{*}},
$$

where $K_{s}=-\left(s_{11}+s_{12}\right) /\left(4 \varepsilon_{0} Q_{12}\right)>0$ is the material parameter governing the strain sensitivity of the reciprocal permittivity. ${ }^{29}$ Equation (4) predicts the film permittivity to increase drastically as the negative misfit strain approaches the critical value $S_{m}^{*}$. At positive misfit strains, the out-ofplane dielectric response should decrease monotonically with increasing strain up to $S_{m}=S_{m}^{* *}$.

The Curie-Weiss-type law described by Eq. (4) implies that the inverse of the film capacitance per unit area, $c_{f}^{-1}$ $=t /\left(\varepsilon_{0} \varepsilon_{33}\right)$, is a linear function of the misfit strain. When this strain is a thickness-independent quantity, the reciprocal capacitance is directly proportional to the film thickness $t$ so that the intercept $c_{f}^{-1}(t \rightarrow 0)$ turns to zero. However, this particular situation takes place only in the range of small thicknesses $t \leq t_{c}$, where the plastic relaxation of the film strains is absent. As soon as the film thickness exceeds a critical value $t_{c}$, above which the formation of misfit dislocations in an epitaxial system is energetically favorable, ${ }^{25}$ the strain $S_{m}$ becomes thickness dependent. According to Eq. (4), this dependence changes the film permittivity and modifies variation of $c_{f}^{-1}$ with the film thickness.

The misfit strain at $t \leq t_{c}$ is equal to the nominal "mismatch" strain $S_{m}^{0}=\left(b-a_{0}\right) / a_{0}$ in an epitaxial couple, which is defined by the difference between the lattice constant $a_{0}$ of a free standing paraelectric film and the in-plane lattice parameter $b$ of the bottom electrode (usually deformed by a thick substrate). Restricting our analysis to epitaxial systems with a small density $\rho \ll 1$ of misfit dislocations situated near the interface, we can evaluate the misfit strain at $t>t_{c}$ as $S_{m}$ $=\left(b^{*}-a_{0}\right) / a_{0}$, where $b^{*} \cong b(1-\rho)$ is the effective lattice parameter of the bottom electrode. ${ }^{26}$ The formation of a dislocation ensemble at the epitaxial interface starts during the film growth and involves thermally activated nucleation and motion of dislocations in the crystal lattice. The dislocation density $\rho$ increases with time, tending to an equilibrium value $\rho^{*}$, which depends mainly on the film thickness and the nominal misfit strain $S_{m}^{0}{ }^{25,26}$ At the growth temperature $T_{g}$ $>T_{c}$, the density of misfit dislocations in an epitaxial system may be estimated as $\rho\left(T_{g}\right) \cong \eta S_{m}^{0}\left(T_{g}\right)\left(1-t_{c} / t\right)$, where the factor $\eta<1$ allows for the fact that usually the equilibrium density $\rho^{*}$ is not reached due to kinetic limitations. ${ }^{14}$ Since Peierls barriers for the dislocation glide are rather high in perovskites, the dislocation density is expected to remain 
practically unchanged during the cooling from the growth temperature: $\rho(T) \cong \rho\left(T_{g}\right)$. Hence, the thickness dependence of the misfit strain at $t>t_{c}$ can be written as

$$
S_{m}(t, T) \cong S_{m}^{\infty}(T)+\eta S_{m}^{0}\left(T_{g}\right) \frac{t_{c}}{t},
$$

where $S_{m}^{\infty}(T)=S_{m}^{0}(T)-\eta S_{m}^{0}\left(T_{g}\right)$ represents the strain in a very thick epitaxial film $\left(t \gg t_{c}\right)$. It should be noted that the misfit strain $S_{m}^{\infty}$ vanishes at $T_{g}$ under equilibrium conditions $(\eta$ =1) but generally differs from zero at $T<T_{g}$. In the first approximation, the temperature dependence of $S_{m}^{\infty}$ may be described by a linear relation

$$
S_{m}^{\infty}(T) \cong(1-\eta) S_{m}^{0}\left(T_{g}\right)+\left(\alpha_{b}-\alpha_{0}\right)\left(T-T_{g}\right),
$$

where $\alpha_{b}$ and $\alpha_{0}$ are the thermal expansion coefficients of the substrate and the paraelectric film, respectively.

The substitution of Eq. (5) into Eq. (4) yields the following relation for the out-of-plane permittivity of a paraelectric film

$$
\frac{1}{\varepsilon_{33}(t, T)}=\frac{1}{\varepsilon_{33}^{\infty}(T)}+\frac{\eta S_{m}^{0}\left(T_{g}\right)}{K_{s}} \frac{t_{c}}{t},
$$

where $\varepsilon_{33}^{\infty}(T)=K_{s} /\left[S_{m}^{\infty}(T)-S_{m}^{*}(T)\right]$ is the permittivity of a very thick strained paraelectric film $\left(t \gg t_{c}\right)$. Equation (7) shows that, depending on the sign of $S_{m}^{0}\left(T_{g}\right)$, the film permittivity either increases or decreases with increasing thickness. Accordingly, the reciprocal capacitance at $t>t_{c}$ becomes

$$
\frac{1}{c_{f}}=\frac{t}{\varepsilon_{0} \varepsilon_{33}^{\infty}}+\frac{\eta S_{m}^{0}\left(T_{g}\right) t_{c}}{\varepsilon_{0} K_{s}} .
$$

It can be seen that the extrapolation of $c_{f}^{-1}$ to zero thickness gives a finite intercept caused by the strain relaxation. Remarkably, when the in-plane film strains at $T_{g}$ are compressive $\left(S_{m}^{0}<0\right)$, the intercept $c_{f}^{-1}(t \rightarrow 0)$ is negative. On the other hand, in the case of tensile strains $\left[S_{m}^{0}\left(T_{g}\right)>0\right]$ the thickness dependence of $c_{f}^{-1}$ has a positive intercept.

\section{B. Ferroelectric regime}

Consider now the dielectric behavior displayed by epitaxial films in the ferroelectric state. We first discuss the range of relatively large negative misfit strains, where the tetragonal $c$ phase with the out-of-plane spontaneous polarization $\left(P_{1}=P_{2}=0, P_{3} \neq 0\right)$ represents the energetically most favorable state. Near the second-order phase transition, where the $P^{4}$ approximation is valid, the dependence of the film permittivity $\varepsilon_{33}$ on the misfit strain takes the form ${ }^{14}$

$$
\varepsilon_{33}\left(S_{m}\right)=\frac{K_{s c}}{S_{m}^{*}-S_{m}},
$$

where $S_{m}<S_{m}^{*}$ and $K_{s c}=K_{s} / 2$. Using Eqs. (5) and (9), we obtain the relation

$$
\frac{1}{\varepsilon_{33}(t, T)}=\frac{1}{\varepsilon_{33}^{\infty}(T)}-\frac{\eta S_{m}^{0}\left(T_{g}\right)}{K_{s c}} \frac{t_{c}}{t},
$$

which defines the dielectric response of the $c$ phase as a function of the film thickness at $t>t_{c}$. It can be seen that the thickness-dependent term in Eq. (10) differs from that in Eq.
(7) by the opposite sign and by a two times larger magnitude. The parameter $\varepsilon_{33}^{\infty}(T)=K_{s c} /\left[S_{m}^{*}(T)-S_{m}^{\infty}(T)\right]$ involved in Eq. (10) usually represents the permittivity of a very thick ferroelectric film. However, the misfit strain at $t \gg t_{c}$ may relax to a value at which the inequality $S_{m}^{\infty}<S_{m}^{*}$ is not valid anymore so that $\varepsilon_{33}^{\infty}$ becomes negative. In this situation, which may take place at $T>\theta$, the parameter $\varepsilon_{33}^{\infty}$ has no physical meaning since at $t \rightarrow \infty$ the film would be in the paraelectric state, which is described by another formalism (see the preceding subsection).

According to Eq. (10), the thickness dependence of the reciprocal capacitance can be written in the discussed case as

$$
\frac{1}{c_{f}}=\frac{t}{\varepsilon_{0} \varepsilon_{33}^{\infty}}-\frac{\eta S_{m}^{0}\left(T_{g}\right) t_{c}}{\varepsilon_{0} K_{s c}} .
$$

Since the $c$ phase can form only at negative misfit strains $S_{m}<S_{m}^{*}(T>\theta),{ }^{24,28}$ the strain $S_{m}^{0}\left(T_{g}\right)$ involved in Eq. (11) usually must be also negative. Therefore, in the ferroelectric regime the intercept $c_{f}^{-1}(t \rightarrow 0)$ normally is expected to be positive. In principle, it may be possible to observe the dependence $c_{f}^{-1}(t)$ with a negative intercept below the transition temperature $T_{c}$ as well. Equations (5) and (6) show that this special situation occurs when the thermal expansion coefficient $\alpha_{b}$ of the substrate is considerably larger than $\alpha_{0}$ so that the negative strain change $\left(\alpha_{b}-\alpha_{0}\right)\left(T_{c}-T_{g}\right)$ during the film cooling overcompensates the initial small positive strain $S_{m}\left(t, T_{g}\right)$ in the whole thickness range $t_{\min } \leq t \leq t_{\max }$ studied experimentally.

In the range of large positive misfit strains, the ferroelectric phase transition must lead to the formation of an in-plane polarization state $\left(P_{1} \neq 0, P_{2} \neq 0, P_{3}=0\right)$ in an epitaxial film. ${ }^{24,28,30}$ For such ferroelectric state (e.g., orthorhombic $a a$ phase), the permittivity calculated in the $P^{4}$ approximation obeys the Curie-Weiss-type law of the form

$$
\varepsilon_{33}=\frac{K_{s a}}{S_{m}-\widetilde{S}_{m}^{*}},
$$

where $S_{m}>\widetilde{S}_{m}^{*}(T)$ and $K_{s a}^{-1}$ is the strain sensitivity of the film reciprocal permittivity. ${ }^{29}$ Equation (12) is similar to Eq. (4), but the parameters $K_{s a}$ and $\widetilde{S}_{m}^{*}(T)$ involved here differ from $K_{s}$ and $S_{m}^{*}$. In particular, $\widetilde{S}_{m}^{*}$ represents the critical misfit strain at which the in-plane polarization state loses its stability against the appearance of the out-of-plane polarization $P_{3}$. From Eqs. (5) and (12) it follows that the dependence $c_{f}^{-1}(t)$ remains linear, being described by a relation similar to Eq. (8). Since $K_{s a}>0$ (Ref. 29) and the strain $S_{m}^{0}\left(T_{g}\right)$ should be normally also positive to ensure the formation of in-plane polarization state, the intercept $c_{f}^{-1}(t \rightarrow 0)$ appears to be positive in the discussed case of a ferroelectric film grown on a tensile substrate.

Well below the transition temperature, the dependence $\varepsilon_{33}\left(S_{m}\right)$ cannot be described by Eq. (9) or (11) anymore since the sixth-order terms in the free-energy expansion become substantial here. Nevertheless, the numerical calculations show that the inverse of the film permittivity, $1 / \varepsilon_{33}$, still varies with the misfit strain almost linearly. Accordingly, the thickness dependence of reciprocal capacitance $c_{f}^{-1}$ may be 
approximated by a linear relation similar to Eq. (11). It should be emphasized, however, that the parameters involved in this relation do not admit of a simple physical interpretation.

In the intermediate range of small positive and negative misfit strains, both the in-plane and out-of-plane polarization components differ from zero, and the strain dependence of the permittivity may be nonmonotonic. ${ }^{28,30}$ Therefore, the thickness dependence of inverse capacitance $c_{f}^{-1}$ is expected to be nonlinear here and so beyond the scope of the present article.

\section{EFFECT OF DEPOLARIZING FIELD ON PERMITTIVITY AND CAPACITANCE}

We proceed now to the analysis of the influence of the depolarizing field on the dielectric response of a ferroelectric capacitor. The depolarizing field appears when the polarization charges $\rho=-\operatorname{div} \mathbf{P}$ existing at the film surfaces are not perfectly compensated for by other charges. Since the electronic screening length in metals is finite, the depolarizing field may be significant even in short-circuited ferroelectric capacitors. $^{21,31}$ To simplify the theoretical description of the depolarizing-field effect, we model the ferroelectric/electrode interfaces by thin low-permittivity dielectric layers. The thickness $d \ll t$ and permittivity $\varepsilon_{d}$ of these layers are taken to be independent of the film thickness $t$. If variations of the ferroelectric polarization near the film surfaces are negligible, the parameters $d$ and $\varepsilon_{d}$ of the interfacial layer can be evaluated as $d=\sqrt{\varepsilon_{m}} l_{\mathrm{TF}}$ and $\varepsilon_{d}=\varepsilon_{m}$, where $l_{\mathrm{TF}}$ and $\varepsilon_{m}$ are the Thomas-Fermi screening length and the lattice dielectric constant of the electrode material, respectively. ${ }^{32}$

Since the depolarizing field is created by the out-ofplane polarization only, we focus on ferroelectric films grown on compressive substrates, which stabilize in the tetragonal $c$ phase $\left(P_{1}=0, P_{2}=0, P_{3} \neq 0\right)$. We assume the film to be in the single-domain state, although the depolarizing field generally favors the formation of $180^{\circ}$ domains. $^{33,34}$ This model enables us to determine the maximum effect of depolarizing field on the dielectric response of a ferroelectric film. Moreover, the application of an electric field stronger than the coercive field makes the film polarization state uniform on the macroscopic scale. This uniform state is characteristic of the films poled prior to the small-signal dielectric measurements. Therefore, it should usually persist at zero direct current (dc) voltage during the measurements of capacitance-voltage loops as well. It should be emphasized that well below the transition temperature the single-domain state remains stable against the transformation into the $180^{\circ}$ domain pattern down to very small film thicknesses. ${ }^{35}$ It is also worth noting that ultrathin $\mathrm{PbTiO}_{3}$ films grown on $\mathrm{SrTiO}_{3}$ were found to be single-domain at room temperature despite the absence of electrodes. ${ }^{36,37}$

The dielectric response in the presence of a depolarizing field can be calculated from the dependence of the out-ofplane polarization $P_{3}$ on the measuring electric field. In turn, this polarization is determined by the nonlinear equation of state of the strained $c$ phase. Assuming the film to be homogeneously polarized and differentiating the film thermodynamic potential, ${ }^{24,38}$ we obtain

$$
2 a_{3}^{*} P_{3}+4 a_{33}^{*} P_{3}^{3}+6 a_{111} P_{3}^{5}+8 a_{1111} P_{3}^{7}+\cdots=E_{3},
$$

where $a_{3}^{*}=a_{1}-2 S_{m} Q_{12} /\left(s_{11}+s_{12}\right), \quad a_{33}^{*}=a_{11}+Q_{12}^{2} /\left(s_{11}+s_{12}\right)$, $a_{1}=(T-\theta) / 2 \varepsilon_{0} C$, and $a_{i j}, a_{i j k}, a_{i j k l}$ are the higher-order dielectric stiffness coefficients at constant stress. The electric field $E_{3}$ in the ferroelectric film can be calculated as a function of voltage $V$ applied to the electrodes using the continuity conditions for the electric displacement $D_{3}=\varepsilon_{0} E_{3}+P_{3}$ and the electrostatic potential at the interfaces. ${ }^{27}$ The relation derived for $E_{3}$ enables us to rewrite Eq. (13) as

$$
\begin{aligned}
2\left[a_{3}^{*}\right. & \left.+\frac{1}{2\left(\varepsilon_{0}+c_{i} t\right)}\right] P_{3}+4 a_{33}^{*} P_{3}^{3}+6 a_{111} P_{3}^{5}+8 a_{1111} P_{3}^{7}+\cdots \\
& =-\frac{c_{i}}{\left(\varepsilon_{0}+c_{i} t\right)} V
\end{aligned}
$$

where $c_{i}=(1 / 2) \varepsilon_{0} \varepsilon_{d} / d$ is the total capacitance density of two interfacial layers. Equation (14) shows that the coefficient of the lowest-order polarization term changes due to existence of a finite interfacial capacitance $c_{i}$. This renormalization indicates that the depolarizing-field effect reduces the stability range of the $c$ phase in the misfit strain-temperature plane. The voltage-dependent term in Eq. (14) is also affected by $c_{i}$, but its change is usually negligible (for realistic values of the parameters involved, $\varepsilon_{d} t \gg d$ so that $c_{i} t \gg \varepsilon_{0}$ ).

Equation (14) determines the film polarization $P_{3}$ $=P_{3}(V)$ at a given voltage $V$. Since the electric displacement $D_{3}$ in the capacitor equals $D_{3}=\left(P_{3}+\varepsilon_{0} V / t\right) c_{i} t /\left(\varepsilon_{0}+c_{i} t\right)$, the total capacitance density $c_{t}(V)=\partial D_{3}(V) / \partial V$ can be calculated via Eq. (14) as well. The calculation gives

$$
c_{t}=\frac{c_{i} t}{\left(\varepsilon_{0}+c_{i} t\right)}\left[\frac{\varepsilon_{0}}{t}+\frac{c_{i}}{1+\left(\varepsilon_{0}+c_{i} t\right) \chi_{33}}\right],
$$

where $\chi_{33}=2 a_{3}^{*}+12 a_{33}^{*} P_{3}^{2}+30 a_{111} P_{3}^{4}+56 a_{1111} P_{3}^{6}+\cdots$ represents the inverse susceptibility $\chi_{33}=\partial E_{3} / \partial P_{3}$ of the ferroelectric film in the capacitor. The depolarizing field affects $\chi_{33}$ via the suppression of the film out-of-plane polarization $P_{3}$. At constant misfit strain, this polarization becomes smaller in thinner films. Hence, the film intrinsic permittivity $\varepsilon_{33}$ must increase with decreasing thickness $t$ within the stability range of the single-domain ferroelectric state. This unusual dependence is illustrated in Fig. 1, where the small-signal permittivity $\varepsilon_{33}(V \rightarrow 0)$ and the spontaneous polarization $P_{s}$ $=P_{3}(V=0)$ of $\mathrm{Pb}\left(\mathrm{Zr}_{0.5} \mathrm{Ti}_{0.5}\right) \mathrm{O}_{3}$ thin films are plotted at some representative values of the interfacial capacitance and misfit strain. ${ }^{39}$ (For clarity of presentation, in this section the misfit strain is taken to be independent of the film thickness.)

We focus now on the thickness dependence of the smallsignal capacitance $c_{t}(V \rightarrow 0)$, assuming that $c_{i} t \gg \varepsilon_{0}$. Since the coefficient $a_{33}^{*}$ is positive for perovskite ferroelectrics, ${ }^{24,38}$ we may first evaluate $c_{t}(t)$ using the $P^{4}$ approximation for the film thermodynamic potential. This approximation yields $P_{s}^{2} \cong-\left[a_{3}^{*}+1 /\left(2 c_{i} t\right)\right] /\left(2 a_{33}^{*}\right)$ and $\chi_{33} \cong-4 a_{3}^{*}$ $-3 /\left(c_{i} t\right)$. Accordingly, the inverse of the total capacitance $c_{t}(V \rightarrow 0)$ can be written as 


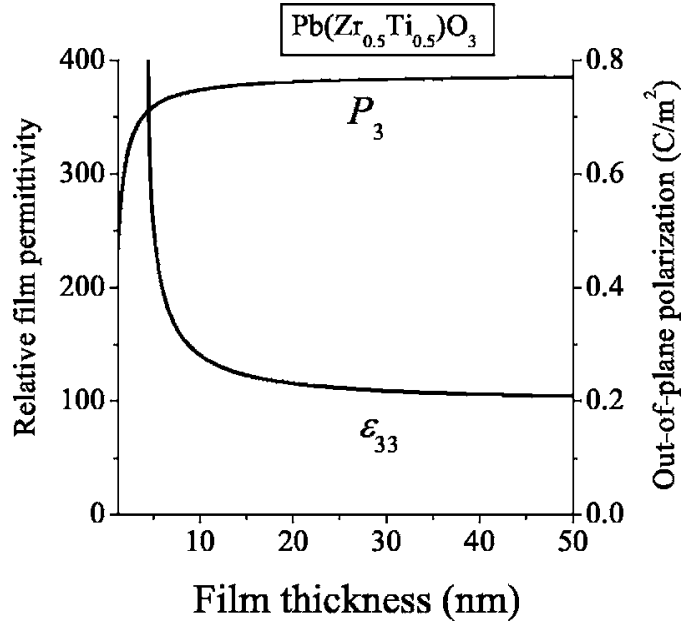

FIG. 1. Thickness dependence of the out-of-plane permittivity $\varepsilon_{33}(V \rightarrow 0)$ and spontaneous polarization $P_{3}(V=0)$ calculated for epitaxial $\mathrm{Pb}\left(\mathrm{Zr}_{0.5} \mathrm{Ti}_{0.5}\right) \mathrm{O}_{3}$ thin films. The temperature equals $25^{\circ} \mathrm{C}$, the interfacial capacitance is taken to be $1 \mathrm{~F} / \mathrm{m}^{2}$, and the misfit strain is $-15 \times 10^{-3}$.

$$
\frac{1}{c_{t}} \cong \chi_{33} t+\frac{1}{c_{i}}=\frac{t}{\varepsilon_{0} \varepsilon_{33}^{\infty}}-\frac{2}{c_{i}},
$$

where $\varepsilon_{33}^{\infty}$ is the relative film permittivity in the absence of the depolarizing-field effect $\left(c_{i} \rightarrow \infty\right)$. Equation (16) demonstrates that the extrapolation of $c_{t}^{-1}$ to zero film thickness gives a negative intercept $c_{t}^{-1}(t \rightarrow 0)=-2 / c_{i}$, in striking contrast to the prediction of the series capacitor model [see Eq. (1)]. This remarkable feature is due to the thickness dependence of the intrinsic dielectric response, which is characteristic of the ferroelectric $c$ phase in the presence of depolarizing field. For the paraelectric phase and the in-plane polarization states, this dependence is absent so that the standard result $c_{t}^{-1}(t \rightarrow 0)=1 / c_{i}$ is recovered. Moreover, when the linear approximation $P_{3}=P_{s}+\chi_{33}^{-1} E_{3}$ is used for the field dependence of polarization, this standard result is erroneously obtained even for the out-of-plane ferroelectric state. This fact explains why only positive values of the parameter $c_{t}^{-1}(t \rightarrow 0)$ were predicted in the recent paper by Mokrý et $a l^{40}$

In the $P^{6}$ and higher-order approximations, the dependence $c_{t}^{-1}(t)$ becomes nonlinear. The numerical calculations, however, show that the nonlinearity is usually negligible when $c_{t}^{-1}(t)$ is plotted over a limited thickness range $t_{\min }$ $\leq t \leq t_{\max }$ only. Hence, the thickness dependence of the reciprocal capacitance may be approximated by a relation $c_{t}^{-1}$ $\cong t /\left(\varepsilon_{0} \varepsilon_{\text {eff }}\right)-\kappa / c_{i}$, which is similar to Eq. (16) but involves two fitting parameters, $\varepsilon_{\text {eff }}$ and $\kappa$. Remarkably, the extrapolation to zero thickness gives a negative intercept different from $-2 / c_{i}$. In the case of $\mathrm{Pb}\left(\mathrm{Zr}_{0.5} \mathrm{Ti}_{0.5}\right) \mathrm{O}_{3}$ films, this difference was found to be relatively small and only weakly dependent on temperature. For the strained $\mathrm{Pb}\left(\mathrm{Zr}_{0.5} \mathrm{Ti}_{0.5}\right) \mathrm{O}_{3}$ capacitor $\left(S_{m}=-15 \times 10^{-3}\right)$ with electrodes made of an elemental metal like $\mathrm{Cu}$ or $\mathrm{Pt}\left(c_{i} \approx 0.2 \mathrm{~F} / \mathrm{m}^{2}\right.$, see Ref. 20$)$, for instance, the parameter $\kappa$ was calculated to be about 2.4 at $25^{\circ} \mathrm{C}$ and about 2.3 at $700{ }^{\circ} \mathrm{C}$.

In contrast, $\mathrm{BaTiO}_{3}$ films were found to have a strong temperature dependence of $\kappa$. Using the $P^{8}$ approximation, which was proposed recently for $\mathrm{BaTiO}_{3}$ crystals, ${ }^{41}$ we cal-

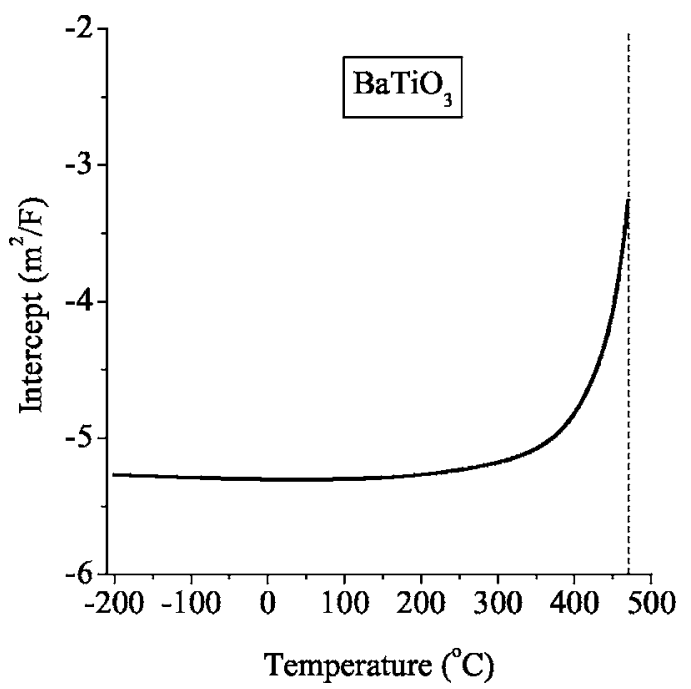

FIG. 2. Temperature dependence of the intercept $c_{t}^{-1}(t \rightarrow 0)$ caused by the depolarizing-field effect. The calculations are performed for ferroelectric $\mathrm{BaTiO}_{3}$ capacitors kept at a fixed misfit strain of $-10 \times 10^{-3}$. The interfacial capacitance is assumed to be $1 \mathrm{~F} / \mathrm{m}^{2}$.

culated the reciprocal capacitance $c_{t}^{-1}(t, T)$ for the thickness range between $t_{\min }=100 \mathrm{~nm}$ and $t_{\max }=1000 \mathrm{~nm}$ at a representative misfit strain $S_{m}=-10 \times 10^{-3}$. ${ }^{42}$ Extrapolating $c_{t}^{-1}$ to zero thickness, we determined the intercept $c_{t}^{-1}(t \rightarrow 0)$ as a function of temperature (see Fig. 2). The plot shown in Fig. 2 demonstrates that the intercept changes rapidly near the critical temperature $T_{\mathrm{cr}} \cong 470{ }^{\circ} \mathrm{C}$, above which the singledomain state becomes unstable at our minimum thickness of $100 \mathrm{~nm}$ (see Sec. IV). Here it falls from $-3.25 / c_{i}$ at $T$ $=470{ }^{\circ} \mathrm{C}$ down to $-4.9 / c_{i}$ at $T=400{ }^{\circ} \mathrm{C}$. Well below $T_{\mathrm{cr}}$, the intercept becomes only weakly dependent on temperature, being about $-5.3 / c_{i}$.

Thus, the apparent interfacial capacitance extracted from the measured dependence $c_{t}^{-1}(t)$ may be negative and temperature-dependent. The calculated values of $c_{t}^{-1}(t \rightarrow 0)$ correspond to strongly polarized films, where the macroscopic remanent polarization $P_{r}$ is close to the spontaneous polarization $P_{s}$. The reduction of the ratio $P_{r} / P_{s}$ is expected to shift the intercept toward zero. In the limiting case of a film having zero average polarization and a dense domain structure, the depolarizing-field effect should be negligible so that the intercept becomes positive and close to $1 / c_{i}{ }^{43}$ Therefore, at some intermediate value of the ratio $P_{r} / P_{s}$ the intercept must turn to zero despite the presence of a finite interfacial capacitance $c_{i}$.

\section{COMPETITION BETWEEN STRAIN AND DEPOLARIZING-FIELD EFFECTS}

When the film thickness exceeds the critical thickness $t_{c}$ for initial generation of misfit dislocations and the misfit strain $S_{m}$ favors the out-of-plane polarization state, both the strain relaxation and the depolarizing field affect the thickness dependence of the spontaneous polarization, dielectric response, and other physical characteristics. This applies, in particular, to the critical temperature $T_{\text {cr }}$, above which the single-domain polarization state becomes unstable with respect to the transformation into the $180^{\circ}$ domain pattern. ${ }^{44}$ 
The lower bound of $T_{\mathrm{cr}}$ is given by the temperature at which the film inverse susceptibility $\chi_{33}=\partial E_{3} / \partial P_{3}$ goes to zero. ${ }^{35}$ In the $P^{4}$ approximation, the condition $\chi_{33}=0$ yields

$$
\begin{aligned}
T_{\mathrm{cr}} \approx & \theta+\frac{4 \varepsilon_{0} C Q_{12}}{\left(s_{11}+s_{12}\right)} S_{m}^{\infty}\left(T_{g}\right) \\
& -\varepsilon_{0} C\left[\frac{3}{2 c_{i}}-\frac{4 Q_{12} t_{c} \eta}{\left(s_{11}+s_{12}\right)} S_{m}^{0}\left(T_{g}\right)\right] \frac{1}{t},
\end{aligned}
$$

where we took into account the thickness dependence of the misfit strain given by Eq. (5) but neglected its weak temperature dependence described by Eq. (6). It can be seen that the thickness dependence of $T_{\mathrm{cr}}$ is determined by the difference between two terms in square brackets, which are involved in Eq. (17). Since the first term is positive whereas the second one must be negative $\left(S_{m}^{0}<0\right.$ and $\left.Q_{12}<0\right)$, the variation of $T_{\text {cr }}$ is governed by the competition of the strain relaxation and the depolarizing-field effect. When the latter prevails, the critical temperature is expected to decrease in thinner films. On the other hand, $T_{\text {cr }}$ will increase with decreasing thickness, if the dominant term in Eq. (17) is associated with the relaxation of compressive misfit strain. The theoretical analysis shows that the temperature $T_{m}$, at which the film has the maximum dielectric response, may also increase or decrease with the film thickness in similarity with the critical temperature $T_{\mathrm{cr}}$. The reduction of $T_{m}$ in thinner films was observed in $\mathrm{SrRuO}_{3} / \mathrm{Ba}_{0.7} \mathrm{Sr}_{0.3} \mathrm{TiO}_{3} / \mathrm{SrRuO}_{3}, \mathrm{Pt} / \mathrm{Ba}_{0.7} \mathrm{Sr}_{0.3} \mathrm{TiO}_{3} / \mathrm{Pt}$, and $\mathrm{Au} / \mathrm{Ba}_{0.5} \mathrm{Sr}_{0.5} \mathrm{TiO}_{3} / \mathrm{La}_{0.5} \mathrm{Sr}_{0.5} \mathrm{CoO}_{3}$ thin-film capacitors. ${ }^{11,15,23}$ At the same time, the dielectric-peak position $T_{m}$ was found to shift to higher temperatures with decreasing thickness in $\mathrm{Pt} / \mathrm{Ba}_{0.7} \mathrm{Sr}_{0.3} \mathrm{TiO}_{3} / \mathrm{SrRuO}_{3}$ and $\mathrm{Au} / \mathrm{Ba}_{0.5} \mathrm{Sr}_{0.5} \mathrm{TiO}_{3} / \mathrm{SrRuO}_{3}$ capacitors. $^{15,22}$

The competition between the strain and depolarizingfield effects also governs the thickness dependence of the film polarization and permittivity at $t>t_{c}$. The reciprocal capacitance calculated in the $P^{4}$ approximation is given by the relation

$$
\frac{1}{c_{t}} \cong \frac{t}{\varepsilon_{0} \varepsilon_{33}^{\infty}}-2\left[\frac{1}{c_{i}}-\frac{4 Q_{12} t_{c} \eta}{\left(s_{11}+s_{12}\right)} S_{m}^{0}\left(T_{g}\right)\right],
$$

where $\varepsilon_{33}^{\infty}$ is the relative permittivity of a thick strained film in the absence of the depolarizing-field effect $\left(t \rightarrow \infty, c_{i} \rightarrow \infty\right)$. Equation (18) demonstrates that the dependence $c_{t}^{-1}(t)$ remains linear, but the intercept is defined now by the algebraic sum of two terms opposite in sign. Evidently, the intercept becomes positive when the contribution due to strain relaxation is larger than the depolarizing-field contribution. The situation reverses in the paraelectric state, where we have $c_{t}^{-1}(t \rightarrow 0) \cong c_{i}^{-1}-4 Q_{12} t_{c} \eta S_{m}^{0}\left(T_{g}\right) /\left(s_{11}+s_{12}\right)$. Remarkably, the intercept here becomes negative when the strain-related effect prevails over the depolarizing-field one.

To investigate the thickness dependence of reciprocal capacitance in a wide temperature range, we performed numerical calculations of $c_{t}^{-1}(t)$ for epitaxial $\mathrm{BaTiO}_{3}$ films in the $P^{8}$ approximation. The variation of the film thickness $t$ was restricted to values between $t_{\min }=100 \mathrm{~nm}$ and $\mathrm{t}_{\max }$ $=1000 \mathrm{~nm}$, and the misfit strain $S_{m}$ was assumed to vary with $t$ in the same way as in $\mathrm{Ba}_{0.7} \mathrm{Sr}_{0.3} \mathrm{TiO}_{3}$ films grown on $\mathrm{SrRuO}_{3}$-covered $\mathrm{SrTiO}_{3}$ (see Ref. 14). The interfacial ca-
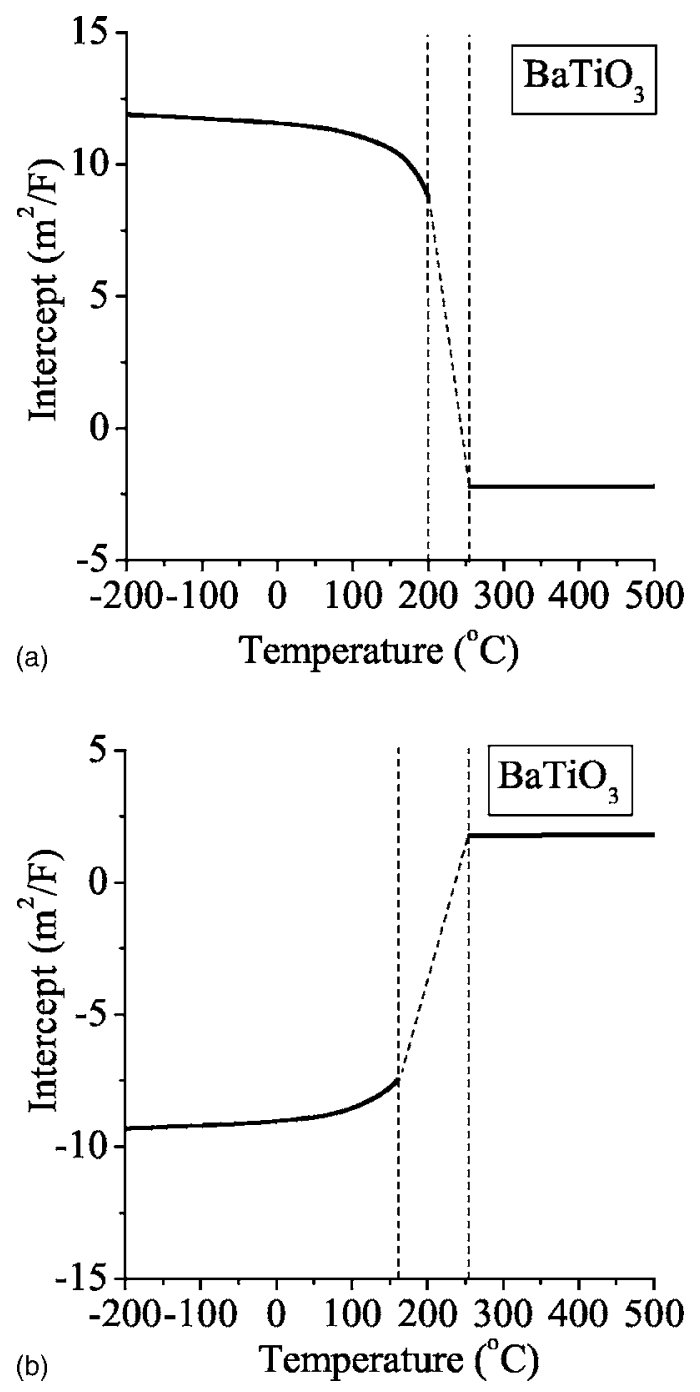

FIG. 3. Temperature dependence of the intercept $c_{t}^{-1}(t \rightarrow 0)$ calculated for $\mathrm{BaTiO}_{3}$ thin-film capacitors with different electrodes. The interfacial capacitance $c_{i}$ is taken to be 1 (a) and $0.2 \mathrm{~F} / \mathrm{m}^{2}$ (b). The thickness-dependent misfit strain $S_{m}$ in the epitaxial film is assumed to be defined by the following set of parameters: $S_{m}^{0}\left(T_{g}\right)=-15 \times 10^{-3}, t_{c}=10 \mathrm{~nm}, \eta=0.7$, and $\partial S_{m} / \partial T$ $=4 \times 10^{-6} \mathrm{~K}^{-1}$.

pacitance $c_{i}$ was taken to be either 1 or $0.2 \mathrm{~F} / \mathrm{m}^{2}$ in order to model electrodes with good and poor screening properties. For the ferroelectric $c$ phase, the dependence $c_{t}^{-1}(t)$ was determined at temperatures not exceeding the minimum critical temperature $T_{\mathrm{cr}}^{\mathrm{min}}=\min \left[T_{\mathrm{cr}}(t)\right]$ calculated from the equation $\chi_{33}(T, t)=0$, since above this temperature the single-domain state may be already unstable. For the same reason, the paraelectric regime was studied only down to the temperature $T_{c} \cong \theta+4 \varepsilon_{0} C Q_{12} S_{m}\left(t_{\text {min }}\right) /\left(s_{11}+s_{12}\right)=255.5^{\circ} \mathrm{C}$, below which the inverse susceptibility $\chi_{33}=2 a_{3}^{*}$ of the paraelectric film becomes negative.

The nonlinearity of the dependence $c_{t}^{-1}(t)$ was proved to be negligible at all studied temperatures, except for a narrow temperature range just below $T_{\mathrm{cr}}^{\mathrm{min}}$, where the thickness dependence of reciprocal capacitance becomes strongly nonlinear and even nonmonotonic. The intercept $c_{t}^{-1}(t \rightarrow 0)$ was then evaluated using the extrapolation procedure and plotted as a function of temperature (see Fig. 3). The plots shown in Fig. 3 demonstrate that at both values of the interfacial ca- 


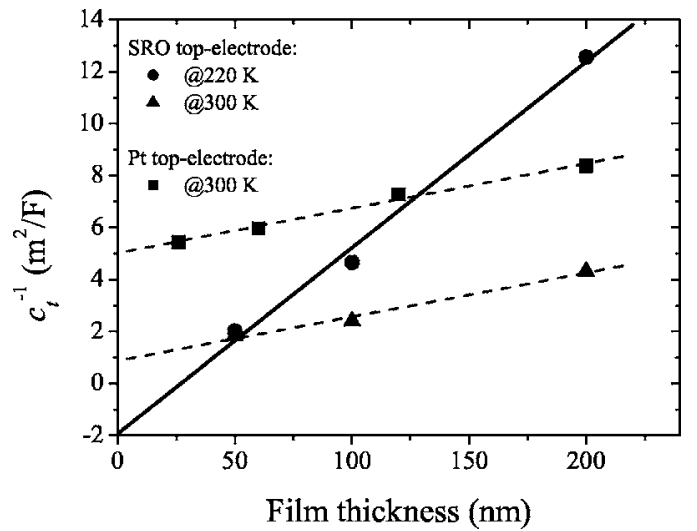

FIG. 4. Representative thickness dependences of the reciprocal capacitance density obtained for $\mathrm{SrRuO}_{3} / \mathrm{Ba}_{0.7} \mathrm{Sr}_{0.3} \mathrm{TiO}_{3} / \mathrm{SrRuO}_{3}$ and $\mathrm{Pt} / \mathrm{Ba}_{07} \mathrm{Sr}_{03} \mathrm{TiO}_{3} / \mathrm{SrRuO}_{3}$ capacitors at different temperatures. The straight lines represent linear fits to the experimental data points.

pacitance the intercept changes sign on crossing the transition region $T_{\mathrm{cr}}^{\mathrm{min}} \leq T \leq T_{c}\left(t_{\mathrm{min}}\right)$. In the case of electrodes with good screening properties $\left(c_{i}=1 \mathrm{~F} / \mathrm{m}^{2}\right)$, the strain effect dominates so that the sign of $c_{t}^{-1}(t \rightarrow 0)$ is positive at low temperatures and negative at high temperatures [Fig. 3(a)]. The decrease of $c_{i}$ down to $0.2 \mathrm{~F} / \mathrm{m}^{2}$, however, is sufficient to reverse the temperature dependence of the intercept [see Fig. 3(b)]. In capacitors with $c_{i}=0.2 \mathrm{~F} / \mathrm{m}^{2}$, therefore, the depolarizing-field effect overrides the influence of strain relaxation.

\section{COMPARISON WITH EXPERIMENT}

We compare the above theoretical predictions with the results of our experimental studies of $\mathrm{SrRuO}_{3} /$ $\mathrm{Ba}_{0.7} \mathrm{Sr}_{0.3} \mathrm{TiO}_{3} / \mathrm{SrRuO}_{3}$ and $\mathrm{Pt} / \mathrm{Ba}_{0.7} \mathrm{Sr}_{0.3} \mathrm{TiO}_{3} / \mathrm{SrRuO}_{3}$ capacitors (termed SRO and Pt samples later). These thin-film capacitors were fabricated on (100)-oriented $\mathrm{SrTiO}_{3}$ single crystals. The perovskite trilayers and bilayers were epitaxially grown in situ by pulsed laser deposition, whereas the Pt top electrode was ex situ deposited on the bilayer samples by dc sputtering. The epitaxial $\mathrm{Ba}_{0.7} \mathrm{Sr}_{0.3} \mathrm{TiO}_{3}$ films were found to be single-crystalline and (001)-oriented (see Refs. 14 and 15 for details on the preparation and characterization of ferroelectric capacitors). The dielectric measurements have been carried out at $10 \mathrm{kHz}$ using an HP4194 impedance analyzer. The capacitance-voltage curves were recorded at different temperatures for samples with the thickness of $\mathrm{Ba}_{0.7} \mathrm{Sr}_{0.3} \mathrm{TiO}_{3}$ layer ranging from 10 to $200 \mathrm{~nm}$. These curves were used to determine the maximum value of the capacitance density $c_{t}$ as a function of the film thickness and temperature. For all $\mathrm{Pt}$ samples, the maximum of the capacitance-voltage curve was found to be shifted to $V_{\max }$ $\approx-0.4 \mathrm{~V}$. This shift can be attributed to an internal electric field caused by the difference in the work functions of two electrodes [5.2 eV for $\mathrm{SrRuO}_{3}$ (Ref. 45) and $5.65 \mathrm{eV}$ for $\mathrm{Pt}$ (Ref. 46)]. Accordingly, the capacitance densities obtained at $V_{\max }=-0.4 \mathrm{~V}$ were used to characterize Pt samples.

The analysis of experimental data demonstrated that the dependence $c_{t}^{-1}(t)$ can be fitted by a linear relation similar to Eq. (1) at all studied temperatures, as demonstrated by representative plots shown in Fig. 4. At room temperature, the
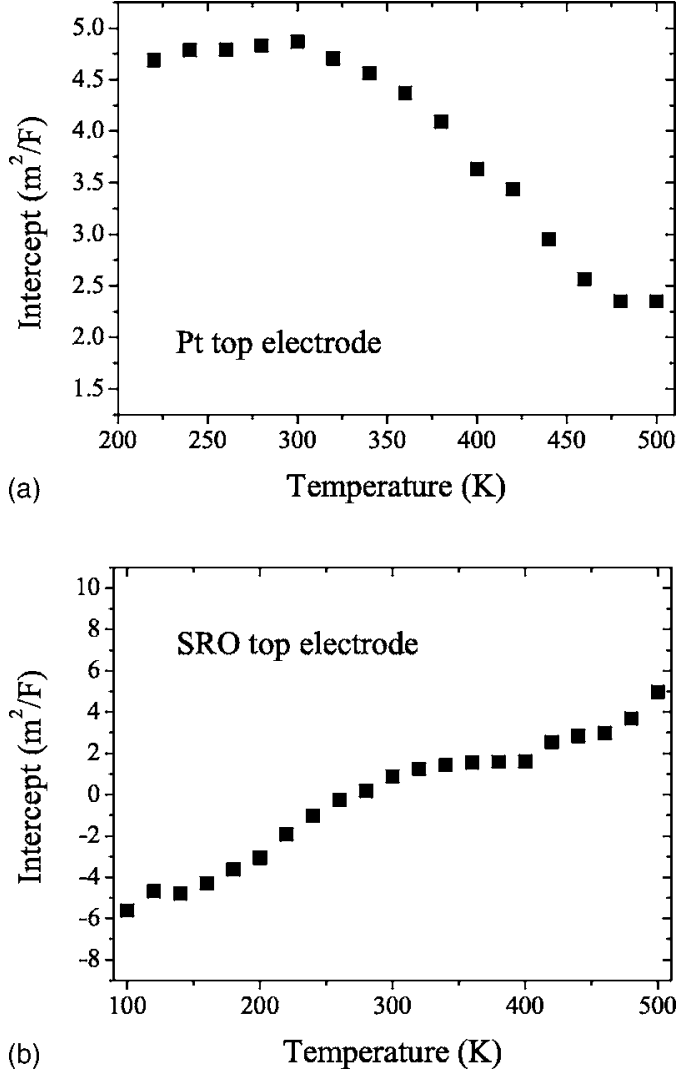

FIG. 5. Temperature dependence of the intercept $c_{t}^{-1}(t \rightarrow 0)$ extracted from the experimental data for $\mathrm{Pt}_{2} / \mathrm{Ba}_{0.7} \mathrm{Sr}_{0.3} \mathrm{TiO}_{3} / \mathrm{SrRuO}_{3}$ (a) and $\mathrm{SrRuO}_{3} / \mathrm{Ba}_{0.7} \mathrm{Sr}_{0.3} \mathrm{TiO}_{3} / \mathrm{SrRuO}_{3}$ capacitors (b).

slope of the linear fit is similar for both sample series, corresponding to the thick-film permittivities $\varepsilon_{33}^{\infty} \approx 6700$ and 5700 for the SRO and Pt samples, respectively. However, the intercept $c_{t}^{-1}(t \rightarrow 0)$ was found to be much larger for the $\mathrm{Pt}$ samples than for the SRO ones. Accordingly, the apparent "interfacial" capacitance density in the case of $\mathrm{SrRuO}_{3}$ top electrode $\left(\approx 1.03 \mathrm{~F} / \mathrm{m}^{2}\right)$ is about five times higher than the value derived for samples with the $\mathrm{Pt}$ top electrode $\left(\approx 0.2 \mathrm{~F} / \mathrm{m}^{2}\right)$. At $T=220 \mathrm{~K}$, the dependence $c_{t}^{-1}(t)$ measured for SRO samples changes drastically (see Fig. 4). The slope of the linear fit strongly increases $\left(\varepsilon_{33}^{\infty} \approx 1000\right)$ and the intercept becomes negative $\left(\approx-2 \mathrm{~m}^{2} / \mathrm{F}\right)$.

Figure 5 shows variations of the measured intercept values in a wide temperature range for both types of capacitors. In the case of $\mathrm{Pt}$ samples, the intercept always remains positive and decreases at high temperatures [see Fig. 5(a)]. Although it does not reach negative values in this range, the observed temperature dependence resembles the theoretical curve shown in Fig. 3(a). On the contrary, the intercept determined for SRO samples changes from positive to negative with decreasing temperature [see Fig. 5(b)]. This trend is similar to the theoretical temperature dependence of $c_{t}^{-1}(t$ $\rightarrow 0$ ) shown in Fig. 3(b). Although the inaccuracy of the intercept obtained from the linear fit increases up to $\pm 2 \mathrm{~m}^{2} / \mathrm{F}$ at $T=100 \mathrm{~K}$ due to the increase of slope, the intercept values remain negative in the whole temperature range below $240 \mathrm{~K}$ even within the experimental error.

Thus, both types of the theoretical temperature dependences shown in Fig. 3 can be observed experimentally. At 
the same time, it is difficult to explain the drastic difference between the dielectric behavior of Pt and SRO samples [Figs. 5(a) and 5(b)] by the competition of the strain and depolarizing-field effects alone. Indeed, as shown experimentally in Ref. 15, these capacitors have practically the same strain state at a given film thickness so that we must assume the interfacial capacitance of the Pt samples to be larger than that of the SRO ones. This assumption, however, contradicts to the theoretical estimates of the capacitance densities associated with the screening space charge in the electrodes, which give $c_{i} \approx 0.27 \mathrm{~F} / \mathrm{m}^{2}$ for the Pt samples and $c_{i} \approx 0.44 \mathrm{~F} / \mathrm{m}^{2}$ for the SRO ones. ${ }^{47}$

The revealed discrepancy between theory and experiment is probably due to one of the following factors, which were not taken into account in our calculations. First, the polarization charges may be additionally screened by the space charge layers forming inside the film owing to the finite conductivity of perovskite ferroelectrics. ${ }^{48,49}$ The theoretical description of the combined internal and external screening of the polarization charges represents a complicated problem. ${ }^{48}$ Here we only note that, for both types of electrode combinations, this effect is expected to increase the interfacial capacitance in comparison with the value determined solely by the screening inside the electrodes.

Second, we ignored the intrinsic size effect on the film permittivity ${ }^{17}$ resulting from local changes of the polarization state near the ferroelectric/electrode interfaces. ${ }^{16}$ The thickness dependence of the permittivity caused by this effect also modifies the intercept $c_{t}^{-1}(t \rightarrow 0) .{ }^{50}$ Remarkably, this dependence is governed by the extrapolation length $\delta$, which may be sensitive to the electrode material. ${ }^{50}$ Therefore, the revealed electrode effect on the dielectric response of $\mathrm{Ba}_{0.7} \mathrm{Sr}_{0.3} \mathrm{TiO}_{3}$ films could be partly due to the polarization variations near the interfaces. According to the phenomenological theory, ${ }^{17}$ the increase of the film permittivity with decreasing thickness, which was observed for SRO samples, can be attributed to the polarization suppression near the film surfaces $(\delta>0)$. At the same time, the polarization enhancement at the $\mathrm{Ba}_{0.7} \mathrm{Sr}_{0.3} \mathrm{TiO}_{3} / \mathrm{Pt}$ interface $(\delta<0)$ is required to explain the thickness dependence of the dielectric response obtained for the Pt samples.

Finally, the dielectric behavior may be also affected by the presence of an internal electric field in the capacitor, being induced, for instance, by the depletion layers ${ }^{50}$ or by the difference in the electrode work functions. If the mean internal field differs from zero, the dielectric peak will be shifted to a higher temperature $T_{m}$ in similarity with the trend caused by an applied electric field. ${ }^{51}$ In asymmetric capacitors like $\mathrm{Pt} / \mathrm{Ba}_{0.7} \mathrm{Sr}_{0.3} \mathrm{TiO}_{3} / \mathrm{SrRuO}_{3}$ ones, therefore, the temperature $T_{m}$ may increase with decreasing film thickness thus providing a positive contribution to the intercept at low temperatures.

\section{CONCLUSION}

In this article, we showed theoretically that the strain relaxation and the depolarizing-field effect usually do not impart pronounced nonlinearity to the thickness dependence of the reciprocal value $c_{t}^{-1}$ of the measured capacitance. This is due to the fact that the variation of the intrinsic film permittivity $\varepsilon_{b}$ with thickness $t$, which is caused by these effects, can be approximated by the relation

$$
\frac{1}{\varepsilon_{b}}=\frac{1}{\varepsilon_{33}^{\infty}}+\frac{\text { const }}{t},
$$

where the constant parameter has the dimension of length. Using Eq. (19), we obtain

$$
\frac{1}{c_{t}}=\frac{t}{\varepsilon_{0} \varepsilon_{33}^{\infty}}+\left(\frac{1}{c_{i}}+\frac{\text { const }}{\varepsilon_{0}}\right)
$$

so that $c_{t}^{-1}$ still varies linearly with the film thickness. However, the simple series capacitor model is not valid anymore because the intercept $c_{t}^{-1}(t \rightarrow 0)$ differs from the reciprocal interfacial capacitance $c_{i}^{-1}$. Since the constant parameter in Eq. (20) is a temperature-dependent quantity which can be positive or negative, the intercept value may strongly vary with temperature, changing its sign on crossing the ferroelectric transition temperature.

Thus, the intercept of the linear thickness dependence of $c_{t}^{-1}$ generally cannot be identified with the actual capacitance density associated with the film/electrode interfaces. In particular, the observation of a negligible intercept at one temperature ${ }^{52}$ does not represent a convincing proof for the absence of "dead" layers in the capacitor. Indeed, such zero intercept may result from the mutual compensation of the positive and negative contributions to $c_{t}^{-1}(t \rightarrow 0)$, but not from the zero interfacial capacitance $c_{i}$. For the better understanding of the influence of interfaces on the dielectric response of ferroelectric capacitors, the intercept has to be measured in a wide temperature range and analyzed carefully.

\section{ACKNOWLEDGMENTS}

The financial support of the Deutsche Forschungsgemeinschaft is gratefully acknowledged. The authors also thank Hermann Kohlstedt for useful discussions.

${ }^{1}$ C. Basceri, S. K. Streiffer, A. I. Kingon, and R. Waser, J. Appl. Phys. 82, 2497 (1997)

${ }^{2}$ S. K. Streiffer, C. Basceri, C. B. Parker, S. E. Lash, and A. Kingon, J. Appl. Phys. 86, 4565 (1999).

${ }^{3}$ N. Yanase, K. Abe, N. Fukushima, and T. Kawakubo, Jpn. J. Appl. Phys., Part 1 38, 5305 (1999)

${ }^{4}$ D. J. Kim, J. Y. Jo, Y. S. Kim, Y. J. Chang, J. S. Lee, J.-G. Yoon, T. K. Song, and T. W. Noh, Phys. Rev. Lett. 95, 237602 (2005).

${ }^{5}$ A. K. Tagantsev, M. Landivar, E. Colla, and N. Setter, J. Appl. Phys. 78, 2623 (1995)

${ }^{6}$ N. A. Pertsev, J. Rodríguez Contreras, V. G. Kukhar, B. Hermanns, H. Kohlstedt, and R. Waser, Appl. Phys. Lett. 83, 3356 (2003).

${ }^{7}$ G. A. Smolenski, and K. S. Rogatschev, Zh. Tekh. Fiz. 27, 1753 (1954);

A. D. Hilton and B. W. Ricketts, J. Phys. D 29, 1321 (1996).

${ }^{8}$ D. E. Kotecki et al., IBM J. Res. Dev. 43, 367 (1999).

${ }^{9}$ P. K. Larsen, J. M. Dormans, D. J. Taylor, and P. J. van Veldhoven, J. Appl. Phys. 76, 2405 (1994).

${ }^{10}$ L. J. Sinnamon, R. M. Bowman, and J. M. Gregg, Appl. Phys. Lett. 78, 1724 (2001)

${ }^{11}$ C. B. Parker, J.-P. Maria, and A. I. Kingon, Appl. Phys. Lett. 81, 340 (2002).

${ }^{12}$ J. McAneney, L. J. Sinnamon, R. M. Bowman, and J. M. Gregg, J. Appl. Phys. 94, 4566 (2003).

${ }^{13}$ W. Y. Park, K. H. Ahn, and C. S. Hwang, Appl. Phys. Lett. 83, 4387 (2003). 
${ }^{14}$ R. Dittmann, R. Plonka, E. Vasco, N. A. Pertsev, J. Q. He, C. L. Jia, S. Hoffmann-Eifert, and R. Waser, Appl. Phys. Lett. 83, 5011 (2003).

${ }^{15}$ R. Plonka, R. Dittmann, N. A. Pertsev, E. Vasco, and R. Waser, Appl. Phys. Lett. 86, 202908 (2005).

${ }^{16}$ R. Kretschmer and K. Binder, Phys. Rev. B 20, 1065 (1979).

${ }^{17}$ W. L. Zhong, B. D. Qu, P. L. Zhang, and Y. G. Wang, Phys. Rev. B 50, 12375 (1994).

${ }^{18}$ C. Zhou and D. M. Newns, J. Appl. Phys. 82, 3081 (1997)

${ }^{19}$ H. Y. Ku and F. G. Ullman, J. Appl. Phys. 35, 265 (1964).

${ }^{20}$ C. T. Black and J. J. Welser, IEEE Trans. Electron Devices 46, 776 (1999).

${ }^{21}$ J. Junquera and Ph. Ghosez, Nature (London) 422, 506 (2003); N. Sai, A. M. Kolpak, and A. M. Rappe, Phys. Rev. B 72, 020101(R) (2005).

${ }^{22}$ L. J. Sinnamon, R. M. Bowman, and J. M. Gregg, Appl. Phys. Lett. 81, 889 (2002)

${ }^{23}$ A. Lookman, R. M. Bowman, J. M. Gregg, J. Kut, M. Dawber, A. Ruediger, and J. F. Scott, J. Appl. Phys. 96, 555 (2004).

${ }^{24}$ N. A. Pertsev, A. G. Zembilgotov, and A. K. Tagantsev, Phys. Rev. Lett. 80, 1988 (1998); Ferroelectrics 223, 79 (1999).

${ }^{25}$ J. Matthews and A. E. Blakeslee, J. Cryst. Growth 27, 118 (1974).

${ }^{26}$ J. S. Speck and W. Pompe, J. Appl. Phys. 76, 466 (1994).

${ }^{27}$ I. P. Batra, P. Würfel, and B. D. Silverman, Phys. Rev. Lett. 30, 384 (1973); Phys. Rev. B 8, 3257 (1973); J. Vac. Sci. Technol. 10, 687 (1973).

${ }^{28}$ V. G. Koukhar, N. A. Pertsev, and R. Waser, Phys. Rev. B 64, 214103 (2001).

${ }^{29}$ N. A. Pertsev, V. G. Koukhar, R. Waser, and S. Hoffmann, Appl. Phys. Lett. 77, 2596 (2000).

${ }^{30}$ V. G. Kukhar, N. A. Pertsev, H. Kohlstedt, and R. Waser, Phys. Rev. B 73, 214103 (2006)

${ }^{31}$ R. R. Mehta, B. D. Silverman, and J. T. Jacobs, J. Appl. Phys. 44, 3379 (1973).

${ }^{32}$ These values of $d$ and $\varepsilon_{d}$ follow from the charge and electric-field distributions inside metallic electrodes calculated in the Thomas-Fermi approximation (see, e.g., Ref. 20).

${ }^{33}$ T. Mitsui and J. Furuichi, Phys. Rev. 90, 193 (1953).

${ }^{34}$ A. M. Bratkovsky and A. P. Levanyuk, Phys. Rev. Lett. 84, 3177 (2000).
${ }^{35}$ N. A. Pertsev and H. Kohlstedt, cond-mat/0603762.

${ }^{36}$ D. D. Fong et al., Phys. Rev. B 71, 144112 (2005).

${ }^{37}$ D. D. Fong et al., Phys. Rev. Lett. 96, 127601 (2006).

${ }^{38}$ N. A. Pertsev, V. G. Kukhar, H. Kohlstedt, and R. Waser, Phys. Rev. B 67, 054107 (2003)

${ }^{39}$ Numerical calculations for $\mathrm{Pb}\left(\mathrm{Zr}_{0.5} \mathrm{Ti}_{0.5}\right) \mathrm{O}_{3}$ films were performed using the $P^{6}$ approximation and the same set of material parameters as in Ref. 38 .

${ }^{40}$ P. Mokrý, A. K. Tagantsev, and N. Setter, Phys. Rev. B 70, 172107 (2004).

${ }^{41}$ Y. L. Li, L. E. Cross, and L. Q. Chen, J. Appl. Phys. 98, 064101 (2005).

${ }^{42}$ For $\mathrm{BaTiO}_{3}$ films, the dielectric stiffness and higher-order stiffness coefficients taken from Ref. 41 and the electrostrictive and elastic constants listed in Ref. 28 were used in the numerical calculations. The interfacial capacitance was assumed to be $c_{i}=1 \mathrm{~F} / \mathrm{m}^{2}$, as expected for electrodes with very good screening properties.

${ }^{43}$ The effects caused by field-induced displacements of $180^{\circ}$ domain walls, which were described in Ref. 40, are expected to be negligible due to high lattice potential barriers in the discussed perovskite ferroelectrics (see Ref. $30)$.

${ }^{44}$ E. V. Chenskiĭ and V. V. Tarasenko, Sov. Phys. JETP 56, 618 (1982).

${ }^{45}$ C. Yoshida, A. Yoshida, and H. Tamura, Appl. Phys. Lett. 75, 1449 (1999).

${ }^{46}$ H. B. Michaelson, IBM J. Res. Dev. 22, 72 (1978).

${ }^{47}$ These values of $c_{i}$ follow from the capacitance densities $c_{\mathrm{Pt}} \approx 0.4 \mathrm{~F} / \mathrm{m}^{2}$ and $c_{\mathrm{SRO}} \approx 0.9 \mathrm{~F} / \mathrm{m}^{2}$ of the $\mathrm{Pt}$ and $\mathrm{SrRuO}_{3}$ electrodes, which can be obtained using the data given in Refs. 4 and 20.

${ }^{48}$ G. M. Guro, I. I. Ivanchik, and N. F. Kovtonyuk, Sov. Phys. Solid State 11, 1574 (1970).

${ }^{49}$ L. Pintilie and M. Alexe, J. Appl. Phys. 98, 124103 (2005).

${ }^{50}$ A. K. Tagantsev, V. O. Sherman, K. F. Astafiev, J. Venkatesh, and N. Setter, J. Electroceram. 11, 5 (2003).

${ }^{51}$ W. J. Merz, Phys. Rev. 91, 513 (1953); H.-M. Christen, J. Mannhart, E. J. Williams, and Ch. Gerber, Phys. Rev. B 49, 12095 (1994); Yu. A. Boikov and T. Claeson, Phys. Solid State 43, 2267 (2001).

${ }^{52}$ Y. S. Kim et al., Appl. Phys. Lett. 88, 072909 (2006). 